
allemande

48-2 | 2016

Les espaces publics des pays germanophones, des espaces publics transnationaux?

\title{
(Re)construire l'espace public germano-suisse. Le journal zurichois Die Tat entre 1945 et 1960
}

\section{Thomas Nicklas}

\section{(2) OpenEdition \\ Journals}

Édition électronique

URL : https://journals.openedition.org/allemagne/391

DOI : $10.4000 /$ allemagne.391

ISSN : 2605-7913

Éditeur

Société d'études allemandes

Édition imprimée

Date de publication : 28 décembre 2016

Pagination : 263-274

ISSN : 0035-0974

Référence électronique

Thomas Nicklas, «(Re)construire l'espace public germano-suisse. Le journal zurichois Die Tat entre 1945 et 1960 », Revue d'Allemagne et des pays de langue allemande [En ligne], 48-2 | 2016, mis en ligne le 28 décembre 2017, consulté le 19 mai 2021. URL : http://journals.openedition.org/allemagne/ 391 ; DOI : https://doi.org/10.4000/allemagne.391 


\section{(Re)construire l'espace public germano-suisse. Le journal zurichois Die Tat entre 1945 et 1960}

- Thomas Nicklas *

«Wir können gar nicht Schweizer sein, ohne uns schlecht oder recht gerade mit den Deutschen auseinanderzusetzen ${ }^{(1)}$.

\section{L'Allemagne et la Suisse dans l'immédiat après-1945: une relation proche, mais compliquée}

Le théologien réformé Karl Barth (1886-1968) fut un acteur transnational entre la Suisse et l'Allemagne. Originaire de Bâle, il a fait des études aux universités allemandes avant d'enseigner comme professeur de théologie à Münster et à Bonn. Opposant farouche au national-socialisme, il est démis de ses fonctions par le régime hitlérien, en 1935, et expulsé d'Allemagne ${ }^{(2)}$. En février 1945, lors d'un discours intitulé Die Deutschen und wir qu'il tient à Zurich, Barth plaide en faveur d'une reprise du dialogue germano-suisse après la fin des hostilités, dès que les circonstances le permettent, puisque l'espace germanophone est, à ses yeux, un espace public commun, formé autour d'une langue et d'une culture partagées ${ }^{(3)}$. C'est la perspective d'un Bâlois, habitant d'une ville frontalière avec l'Allemagne, et d'un protestant suisse dont la confession a connu une longue symbiose culturelle avec le protestantisme d'outreRhin. En même temps, Barth ne peut pas se faire d'illusions sur la profonde rupture que la période du national-socialisme a amenée dans les relations germano-suisses.

* Professeur à l'Université de Reims Champagne-Ardenne, CIRLEP EA 4299.

1 Karl BARTh, Die Deutschen und wir, Zollikon/Zurich, Evangelischer Verlag, 1945, p. 45.

2 Hans Prolingheuer, Der Fall Karl Barth, 1934-1935. Chronik einer Vertreibung, Neukirchen-Vluyn, Neukirchener Verlag, 1984; pour une biographie plus complète, Eberhard BuscH, Karls Barths Lebenslauf, Zurich, TVZ, 2005.

3 Voir en particulier, Eberhard Busch, «Karl Barth und der zwischenkirchliche, karitative und theologische Beitrag der evangelischen Kirchen der Schweiz am deutschen Wiederaufbau », in: Antoine Fleury, Horst Möller, Hans-Peter Schwarz (dir.), Die Schweiz und Deutschland 1945-1961, Munich, Oldenbourg, 2004, p. 229-248. 
Fondée sur un large consensus antitotalitaire, la Suisse des années 1933-1945 s'est rassemblée autour de l'idée de "défense spirituelle» ${ }^{(4)}$ de sa neutralité et de son indépendance, dirigée contre l'emprise des dictatures. Encerclée par les puissances fascistes, après la chute de la France en 1940, elle s'est retrouvée dans la situation d'une «forteresse assiégée» qui, pour assurer sa survie, n’échappa pas aux compromis, ni même aux compromissions, avec les régimes allemand ou italien ${ }^{(5)}$. Pendant les années du «siège», la société suisse a renforcé la tendance au repli sur soi, voire à une isolation linguistique se traduisant par la montée des dialectes alémaniques au détriment de l'emploi de l'allemand standard dans des situations plus formelles, accentuant ainsi une prise de distance intellectuelle par rapport au voisin du nord qui menaçait la liberté et l'identité du pays. Le rejet de l'idéologie national-socialiste, qui allait intrinsèquement de pair avec un certain pangermanisme, par l'écrasante majorité des Suisses, y compris les Suisses germanophones, eut pour conséquence une rupture mentale avec l'Allemagne $^{(6)}$. Or cette anomalie ne pouvait pas durer au-delà de la fin de la guerre et de la chute de l'hitlérisme. À l'instar de Karl Barth, d'autres intellectuels helvétiques comme l'historien et commentateur à la radio suisse Jean Rudolf von Salis ou l'écrivain Max Frisch prirent des initiatives pour renouer le dialogue ${ }^{(7)}$.

Dès la fin de la guerre, en 1945, un dispositif transnational est mis en place pour favoriser un renouveau des relations économiques et culturelles entre la Suisse et les Allemagnes. Le Conseil fédéral, le gouvernement à Berne, coordonne cette politique extérieure portée par des acteurs sociaux pour favoriser le rapprochement entre les nations voisines, tout en accordant une vaste marge de manœuvre aux initiatives de

4 Pour un emploi nuancé du terme, Stefan Andreas Keller, Im Gebiet des Unneutralen. Schweizerische Buchzensur im Zweiten Weltkrieg zwischen Nationalsozialismus und Geistiger Landesverteidigung, Zurich, Chronos, 2009, p. 51-57; Ursula Amrein, Phantasma Moderne. Die literarische Schweiz 1880 bis 1950, Zurich, Chronos, 2007, p. 105-123.

5 Leo Schelbert (dir.), Switzerland under Siege. A neutral nation's struggle for survival, Rockport, 2000. Pour tous les aspects de la politique suisse dans les années 1939-45, qui fit l'objet de longues controverses, la «Commission indépendante d'experts» (Commission Bergier) a fourni une très large documentation: Veröffentlichungen der Unabhängigen Expertenkommission Schweiz - Zweiter Weltkrieg, 25 vol., Zurich, Chronos, 2001/02. Pour un résumé plus récent, Jakob TAnner, Geschichte der Schweiz im 20. Jahrhundert, Munich, Beck, 2015, p. 254-292.

6 Le rapport final de la Commission Bergier a souligné l'immunité de la société suisse, profondément attachée aux valeurs démocratiques, contre l'idéologie nazie: Die Schweiz, der Nationalsozialismus und der Zweite Weltkrieg. Schlussbericht der Unabhängigen Expertenkommission Schweiz - Zweiter Weltkrieg, Zurich, Pendo, 2002, p. 68. Pour une tendance à la rupture dans le domaine de la littérature et du théâtre où la symbiose avait été particulièrement fructueuse, avant 1933, Ursula AmREIN, 'Los von Berlin!' Die Literatur- und Theaterpolitik der Schweiz und das 'Dritte Reich', Zurich, Chronos, 2004. Pour les aspects linguistiques de la défense spirituelle qui souligne le rôle politique et culturel des dialectes alémaniques en Suisse, Eugen Dietн, Die kulturpolitische Bedeutung der schweizerischen Mundarten. Verkürzte Fassung eines am 18. Mai 1942 vor der Stiftung Pro Helvetia gehaltenen Vortrages, Zurich, Schweizerischer Schriftstellerverein, 1943.

7 Hans Ulrich Jost, «Die Haltung schweizerischer Intellektueller gegenüber Nachkriegsdeutschland. Max Frisch und Jean Rudolf von Salis als Beispiel», in: A. Fleury, Die Schweiz und Deutschland (note 3), p. 199-211; Georg Kreis, «Das schweizerische Bild der Deutschen um 1945 », in: G. KreIs, Regina Wecker (dir.), Deutsche und Deutschland aus Schweizer Perspektiven, Basel, Schwabe (=ITINERA, 26), 2007, p. 67-90. 
particuliers et d'organismes humanitaires ou culturels ${ }^{(8)}$. Pour atténuer la misère d'un continent ravagé par le conflit, un regroupement d'œuvres d'entraide suisses, le Don suisse pour les victimes de la guerre (Schweizer Spende für Kriegsgeschädigte), accorde un soutien matériel aux pays marqués par les hostilités, dont l'Allemagne ${ }^{(9)}$. Dans un autre domaine, le Don suisse du livre (Schweizerische Kommission für Bücherhilfe), fondé par l'éditeur bernois Herbert Lang, contribue activement à la reconstruction intellectuelle par le biais de la reconstitution des bibliothèques ${ }^{(10)}$. Interpellé par un député au sujet de l'apport financier de la Confédération à ces dispositifs, le conseiller fédéral et responsable de la politique étrangère suisse, Max Petitpierre, déclare le 24 juin 1946 devant le Conseil national:

«La similitude de langue entre la majorité de notre peuple et le peuple allemand, la communauté de culture qui existait entre la Suisse allemande et l'Allemagne avant que celle-ci se fût abandonnée à un régime qui devait être la négation de toute culture et entraîner le monde entier dans la plus barbare des guerres, ces éléments permettent et doivent même nous engager à mettre nos possibilités intellectuelles à la disposition d'une reconstruction spirituelle et morale de l'Allemagne, dans son propre intérêt et dans celui de l'Europe » ${ }^{(11)}$.

L'accent est mis, par ce porte-parole officiel de la Confédération, sur l'importance d'une reconstruction d'abord intellectuelle en Allemagne, soutenue par des forces de la société civile en Suisse. Il n'est pas question d'un engagement financier de la Confédération ni d'une intervention directe d'acteurs étatiques suisses en Allemagne. Le gouvernement à Berne fait largement appel à l'initiative privée pour débloquer les rapports germano-suisses. Lorsque l'Allemagne fédérale recouvre sa souveraineté diplomatique, en 1951, on voit se nouer des relations officielles entre Berne et Bonn, mais d'importants problèmes restent à l'agenda, dont le règlement de contentieux financiers autour des dettes du Troisième Reich et des avoirs allemands en Suisse, saisis par le Conseil fédéral en février 1945, à l'instigation des États-Unis ${ }^{(12)}$. Pour (re)construire un espace public commun de part et d'autre du Rhin supérieur, dans l'après-1945, il importe aussi de mettre un terme à l'asymétrie des rapports helvéto-allemands, déséquilibrés entre la perception d'un modèle démocratique helvétique d'une part et d'une

8 Un connaisseur des archives suisses et allemandes de cette période parle d'un encadrement officiel d'une politique extérieure sociétale au service d'un rapprochement germano-suisse («einer zwar staatlich koordinierten, aber der Erscheinungsform nach gesellschaftlichen Außenpolitik für die von ihr [i.e. la Confédération] angestrebte Wiederannäherung». Markus Schмiтz, Westdeutschland und die Schweiz. Die Neuformierung der bilateralen Beziehungen 1945-1952, Zurich, NZZ Verlag, 2003, p. 520).

9 Voir surtout Jean-Claude FAVEz, «Le Don suisse et la politique étrangère», in: Barbara Roth-LochNer, Marc Neuenschwander, François Walter (dir.), Des archives à la mémoire. Mélanges d'histoire politique, religieuse et sociale offerts à Louis Binz, Genève, Société d'histoire et d'archéologie, 1995, p. 325-339.

10 Marcus Schmitz, «Die humanitäre und kulturelle Deutschlandhilfe der Schweiz nach dem Zweiten Weltkrieg», in: A. Fleury, Die Schweiz und Deutschland (note 3), p. 213-228, ici p. 221.

11 Citation dans M. Schмiтz, Westdeutschland und die Schweiz nach dem Krieg (note 8), p. 199.

12 Ilse Dorothee Pautsch, "Altschulden und Neubeginn. Die "Clearingmilliarde" und die Aufnahme diplomatischer Beziehungen zwischen der Bundesrepublik Deutschland und der Schweiz", in: A. Fleury, Die Schweiz und Deutschland (note 3), p. 17-29; M. Schmitz, Westdeutschland und die Schweiz (note 8), p. 465-518. 
rupture civilisationnelle dont l'Allemagne, d'autre part, fut la seule responsable. Karl Barth, dans son discours déjà cité de février 1945, met en garde ses concitoyens contre le danger de prodiguer avec condescendance des conseils aux voisins du nord:

«Nun sind wir Schweizer aber von Natur eine pädagogische Nation und die Gefahr ist groß, daß wir den Deutschen gegenüber [...] vor allem aufs Katheder steigen wollen könnten. Das wäre aber ein großes Unglück» ${ }^{(13)}$.

Il fallait œuvrer pour l'intercompréhension au sein des pays d'expression allemande, pour (ré)intégrer l’Allemagne dans une communauté européenne des esprits. Tel fut aussi le projet d'un journal paraissant à Zurich, à savoir le quotidien Die Tat.

\section{Germaniste et germanophile: Erwin Jaeckle et Die Tat}

«Sie können bei mir als selbstverständlich voraussetzen, dass auch ich auf jede Weise gern germanophil bin, indem ich ausserordentlich stark das Bewusstsein habe, dem deutschen Kulturkreis anzugehören », écrit Erwin Jaeckle au mois de février 1943 dans une lettre adressée à Gustav Däniker (1896-1947), un ancien officier suisse licencié de l'armée après avoir plaidé en faveur de la collaboration avec l'Allemagne hitlérienne, en $1941^{(14)}$. Contrairement à Däniker, Jaeckle insiste sur une séparation stricte entre l'idéologie national-socialiste et la culture allemande. Malgré son attachement à cette dernière, il rejette toute compromission avec la dictature qui menace l'indépendance de la Confédération. Le germaniste Jaeckle qui a soutenu une thèse de doctorat consacrée à l'écrivain contemporain Rudolf Pannwitz (1881-1969) à l'université de Zurich, en 1937, va entamer une carrière de journaliste qui devrait lui permettre de cultiver, dans ses moments libres, son amour pour la poésie ${ }^{(15)}$. Début 1943, à l'âge de 33 ans, Erwin Jaeckle devient le rédacteur en chef d'un journal qui occupe une place exceptionnelle dans le paysage médiatique suisse: Die Tat. Le quotidien paraissant à Zurich dépend financièrement de la coopérative Migros, créée par l'entrepreneur zurichois Gottlieb Duttweiler (1888-1962) ${ }^{(16)}$. Pendant la crise économique des années 1930, Duttweiler a dû affronter toutes sortes de monopoles et de corporations, la face cachée du capitalisme helvétique, pour assurer la réussite de son entreprise. Dans sa lutte contre les trusts qui tentaient d'empêcher la restructuration dans le domaine de la grande distribution qu'il envisageait, Duttweiler fonda un parti politique, l'Alliance des Indépendants (Landesring der Unabhängigen). Le parti qui put envoyer sept députés au Conseil national, en 1935, se dota d'un hebdomadaire, transformé en quotidien en 1939. Puisque conçu comme vitrine des idées économiques et politiques de son fondateur, Die Tat va rester

13 «Nous autres Suisses, nous sommes une nation pédagogique par nature et le danger existe que nous montions à la tribune pour sermonner les Allemands. Or ce serait un grand malheur.» K. BARTH, Die Deutschen und wir (note 1), p. 23.

14 «Je vous assure que je suis aussi largement germanophile, puisque j'ai fortement l'idée d'appartenir au monde culturel germanophone." Erwin JAECKLE, Erinnerungen an 'Die Tat' 1943-1971, Zurich, Hans Rohr, 1989, p. 30. Pour Däniker, Therese Keller, Oberst Gustav Däniker. Aufstieg und Fall eines Schweizer Berufsoffiziers, Zurich, Thesis, 1997.

15 Erwin JaEckle, Die Lebenslinie: eine seltsame Biographie, Stäfa ZH, Gut, 1993.

16 Ernst Bollinger, La presse suisse: structure et diversité, Berne, Herbert Lang, 1976, p. 56; Alfred A. HÄsler, L'aventure Migros. 60 ans d'une idée jeune, Lausanne, Coopératives Migros, 1985, p. $229-231$. Pour Duttweiler, Curt Riess, Gottlieb Duttweiler. Eine Biographie (1959), Zurich, Europa-Verlag, 2011. 
largement déficitaire, faute de recettes publicitaires, il devra être subventionné par les coopératives Migros. Journal politique qui soutient avec ferveur l'indépendance du pays contre les ingérences étrangères, Die Tat peut conquérir une place respectable sur le marché de la presse suisse, pendant les années de la guerre 1939-1945.

Travailler pour et avec l'entrepreneur dynamique et parfois impulsif Gottlieb Duttweiler n'est certes pas une tâche facile, mais le patron de la Migros laisse une importante marge de manœuvre aux collaborateurs à qui il fait confiance, et dont le rédacteur en chef de Die Tat sait tirer parti. Dès la fin de la guerre, Erwin Jaeckle profite de sa position pour défendre les intérêts de la «vraie Allemagne», telle qu'il la conçoit, cette Allemagne que le national-socialisme a renversée, à la suite de la prise de pouvoir par Hitler ${ }^{(17)}$. Dans l'immédiat après-guerre, Jaeckle prend position dans les débats menés en Suisse sur la restructuration de l'Europe qui, selon lui, doit nécessairement accorder un rôle important aux nations vaincues. Début 1947, il lance un sondage auprès d'universitaires, d'hommes politiques et d'écrivains en Europe et en Amérique pour connaître leur conception de la future Allemagne et pour sensibiliser, en même temps, le public helvétique aux problèmes de ses voisins allemands ${ }^{(18)}$. Malgré les restrictions administratives et matérielles dans un pays partagé en zones d'occupation, Jaeckle n'hésite pas à entreprendre des voyages. Au mois de septembre 1947, il participe aux premières assises de la nouvelle presse démocratique dans la zone américaine qui se tiennent à Cobourg, en Bavière ${ }^{(19)}$. En coopération avec les rédacteurs en chef des journaux allemands réunis à Cobourg, il prend une initiative pour la liberté de la presse, à l'encontre des forces d'occupation. Jaeckle publie dans Die Tat des articles très critiques à l'égard des Alliés et donc susceptibles d'être censurés. Il réalise une manœuvre judicieuse en faveur de la liberté, puisque ces organes de presse de la zone américaine ont le droit de reproduire tels quels des textes déjà parus à l'étranger ${ }^{(20)}$. Il organise aussi un échange de journalistes entre Zurich et Hambourg et participe à une Table ronde, organisée en février 1949 par la rédaction du Münchner Merkur, où il s'agit d'analyser l'hypothèse d'un nouveau nationalisme en Allemagne, qui se manifesterait en réaction au démantèlement de l'industrie allemande mise en œuvre par les Alliés. Il s'exprime en faveur de l'abandon des concepts nationalistes qui ont envenimé l'avant-guerre et préconise que l'Allemagne pacifiée et démocratisée retrouve sa place parmi les grandes puissances européennes ${ }^{(21)}$. Les idées de Jaeckle, qui visent à favoriser les échanges transnationaux entre la nouvelle République fédérale et la Suisse, sont réunies dans des suppléments spéciaux dans Die Tat, dédiés aux sujets allemands, dont «Die Zukunft Deutschlands» (L'Avenir de l’Allemagne) en août 1950, dans lesquels s'expriment divers hommes politiques, universitaires et industriels ${ }^{(22)}$; «Deutsche Einheit» (Unité allemande) en novembre 1950 ou

17 «...] ich sei von der ersten Stunde an hart gegen den Nationalsozialismus angetreten, um mir damit glaubwürdig das Recht zu sichern, nach dem Zusammenbruch für das solange verschüttete wahre Deutschland eintreten zu können.» E. JAECKLE, Erinnerungen an 'Die Tat' (note 14), p. 80.

18 Le sondage est lancé dans le n 12/10 (1947), p. 12, à la date du 11 janvier 1947 sous l'intitulé «Was die Welt von Deutschland hält».

19 E. JAECKLE, Erinnerungen an 'Die Tat' (note 14), p. 80-82.

20 Ibid., p. 82.

21 Ibid., p. 116-121.

22 Die Tat, 15 (1950), n² 230 du 25.08.1950. 
encore «Deutscher Osten» en avril $1953^{(23)}$, supplément consacré aux territoires de l'Est placés sous administration polonaise ou soviétique. Pour Jaeckle, la finalité principale, l'unité de l'Europe sous la forme d'une confédération des États ne peut être atteinte que par une intégration culturelle de nations européennes: «Zusammengehörigkeiten des Geistes fordern Zusammenschlüsse des Alltags » ${ }^{(24)}$.

Même si la Suisse n'a pas encore entamé de relations diplomatiques avec l'Allemagne fédérale, Jaeckle n'hésite pas à envoyer dès 1949 un correspondant permanent de son journal à Bonn ${ }^{(25)}$. C'est l'un des premiers journalistes étrangers qui se fixent durablement dans cette petite ville rhénane, car tout s'y inscrit, pour l'instant, dans la logique des solutions provisoires. Il s’agit de Fritz René Allemann (1910-1996), ancien correspondant de Die Tat à Londres et à Paris et qui a dirigé la rédaction de politique étrangère du journal à Zurich. Simultanément, le jeune Herbert Lüthy (1918-2002), l'une des plumes les plus prometteuses de la Confédération, devient correspondant à Paris ${ }^{(26)}$. On voit bien que Jaeckle a le talent de constituer une équipe de collaborateurs éminents et loyaux. Selon les idées préconisées par Gottlieb Duttweiler et son journal, une démocratie directe comme celle de la Suisse a besoin de citoyens bien informés. Par conséquent, il importe de présenter au public helvétique les nouvelles politiques et de lui fournir des commentaires aussi lucides que possible ${ }^{(27)}$. Lüthy et Allemann proposeront de très fines analyses de l'évolution en France et en Allemagne ${ }^{(28)}$. Contrairement à Lüthy qui rentrera en Suisse, en 1958, pour occuper un poste de professeur d'histoire moderne à École polytechnique fédérale de Zurich, Fritz René Allemann restera en Allemagne où il servira d'intermédiaire intellectuel entre les deux pays.

\section{Le profil d'un journaliste transnational à Bonn: Fritz René Allemann}

À peine arrivé en République fédérale, le journaliste suisse s’attribue le rôle de médiateur et d'interprète entre les deux cultures, suisse (alémanique) et (ouest-)allemande. Né à Bâle dans une famille d'origine alsacienne, naturalisée suisse en 1927 seulement, il a vécu à Berlin entre 1930 et 1932, où il s'est familiarisé avec les problèmes de la politique allemande dans la phase terminale de la République de Weimar ${ }^{(29)}$. Depuis

23 Die Tat, 15 (1950), $\mathrm{n}^{\circ} 300$ du 04.11.1950; 18 (1953), nº 92 du 03.04.1953.

24 «Les liens intellectuels vont de pair avec des alliances dans la vie quotidienne.» E. JAECKLE, Erinnerungen an 'Die Tat' (note 14), p. 131.

25 Ibid., p. 116.

26 Voir l'introduction biographique du premier volume des Euvres complètes d'Herbert Lüthy: Peter Wegelin, «Einleitung», in: Urs Bitterli, Irene Riesen (éd.), Herbert Lüthy. Gesammelte Werke, vol. I, Zurich, Verlag NZZ, 2002, p. XVII-XXX; pour les activités journalistiques de Lüthy dans l'immédiat après-guerre, Joachim Gmenling, Kritik des Nationalsozialismus und des Sowjetkommunismus in der Zeitschrift 'Der Monat', thèse de l'Université de Hambourg, 2010, p. 356-360.

27 Voir surtout Edgar Bonjour, La neutralité suisse. Synthèse de son histoire, Neuchâtel, Baconnière, 1979, p. 161-163.

28 Herbert Lüтнy, Frankreichs Uhren gehen anders, Zurich, Europa-Verlag, 1954 (la traduction française: À l'heure de son clocher: essai sur la France, Paris, Calmann-Lévy, 1955).

29 Pour la biographie d’Allemann, voir Thomas NickLAs, «'Bonn ist nicht Weimar'. Fritz René Allemann et la création d'un lieu commun », in: Mechthild Coustillac, Jacques LAJARrige, Hilda Inderwildi (dir.), Entre ombres et lumière. Voyages en pays de langue allemande, Toulouse, Presses universitaires du Midi, 2016 (à paraître). 
son poste d'observation à Bonn où il s'installe en 1949, il adopte le programme de Die Tat d'être un forum transnational facilitant les échanges entre deux pays étroitement liés, mais avec des références culturelles souvent divergentes. Lors d'une conférence radiodiffusée en mars 1951 par l'émetteur de Hambourg de la radio NWDR (Nordwestdeutscher Rundfunk), Allemann explique aux auditeurs qu'il veut travailler comme «interprète» entre les sociétés allemande et suisse, en rendant accessibles à ses lecteurs helvétiques les débats tenus en République fédérale, les discours qu’ils comprennent certes linguistiquement, mais dont le contenu leur échappe en grande partie puisque les repères socioculturels diffèrent. Il veut, précise-t-il, faire parler l'Allemagne aux Suisses et traduire ses propos dans le «dialecte mental» de ses compatriotes ${ }^{(30)}$. Les réflexions d'Allemann sur les relations germano-suisses tournent autour de l'idée de diversité linguistique, au premier abord peu évidente pour la Suisse germanophone, sur fond d'une différence mentalitaire profonde ${ }^{(31)}$. La langue du Troisième Reich qui continue de marquer les textes officiels et les discours quotidiens dans l'Allemagne de 1950, rebute les visiteurs suisses en République fédérale et rend encore plus difficile l'intercompréhension entre les deux nations ${ }^{(32)}$. La conception d'une communauté linguistique de part et d'autre du Haut-Rhin, fondamentale pour les Allemands, paraît secondaire et comme une fortuité historique pour les Suisses. Pour sa part, le journaliste Allemann veut contribuer à la compréhension entre les deux peuples. Il est conscient que la force des stéréotypes s'oppose à un rapprochement intellectuel. Oscillant entre le mépris et l'idéalisation, la plupart des Allemands ont du mal à se situer par rapport à la Confédération. Beaucoup d'entre eux envient leur interlocuteur helvétique, à cause de la neutralité de sa patrie dans les conflits qui divisent l'Europe: "Sie kommen aus der Schweiz? Sie haben es gut!» («Vous êtes suisse? Vous avez de la chance!») ${ }^{(33)}$. Or le discrédit dans lequel sont tombées les aspirations impériales de l’Allemagne après 1945 permet d'ouvrir un nouveau chapitre dans les relations bilatérales. Depuis l'époque

30 Allemann s'attribue comme tâche «die Schweizer überhaupt einmal mit den Gefühlen, den Problemen, den Sorgen, den Hoffnungen des deutschen Volkes bekanntzumachen. Und das kann ich nur tun, wenn ich meine Arbeit als die eines Dolmetschers auffasse, d.h. wenn ich mich bemühe, die deutsche Mentalität in eine Sprache zu übersetzen, die dem Schweizer Leser verständlich tönt und ihm das Gefühl der völligen Fremdheit nimmt. [...] Deutschland fängt an zu sprechen - und dann macht es einem auch viel mehr Freude, das, was es spricht, den Leuten daheim in der Schweiz gleichsam in ihren andersartigen geistigen Dialekt zu übersetzen. » Bibliothèque universitaire de Bâle, NL 304 (Nachlass Fritz René Allemann), G3, tapuscript «Als Auslandskorrespondent in Deutschland » (mars 1951).

31 L'écrivain soleurois Peter Bichsel dira la même chose en d'autres termes: «Ich weiß nicht genau, was hochdeutsche Sätze und Wörter bedeuten, die Mentalität, die hinter ihnen steht, ist mir fremd. » Peter Bichsel, «Wie deutsch sind die Deutschen?», in : Jürg Altwegg, Roger de Weck (dir.), Kuhschweizer und Sauschwaben. Schweizer, Deutsche und ihre Hassliebe, Munich/Vienne, Nagel \& Kimche, 2003, p. 159-175, ici p. 159/160.

32 « Der unvorbereitete Schweizer steht in Deutschland zunächst einmal ziemlich fassungslos vor dem neudeutschen Jargon und der Terminologie, die ihm in Gesprächen, Reden, Zeitungsartikeln und amtlichen Erlassen gegenübertritt und in der sich die Erfahrungen und Eindrücke des Nationalsozialismus und des Krieges auf eine Weise niederschlagen, die das Erlebnis der Fremdheit ganz unvermittelt anhörbar macht. » Fritz René AllemanN, "Gedanken zum deutsch-schweizerischen Verhältnis », Die Tat, 15 (1950), n 230 du 25.08.1950, p. 14.

33 Ibid. 
bismarckienne, le dynamisme de la politique allemande n'a cessé de mettre en cause les équilibres fragiles de la Confédération qui, depuis des siècles, a perfectionné l'art de contrebalancer des intérêts contradictoires. Par contre, la jeune République fédérale, toujours à la recherche de sa place en Europe et dans le monde, reconnaît et respecte les particularités suisses. Dans ce nouveau contexte, les échanges culturels et intellectuels ne sont plus au service d'une politique allemande de grande puissance et la Suisse n'est plus une «forteresse assiégée», obligée de se replier sur son réduit alpin pour défendre son indépendance. Allemann se trouve alors sur un terrain favorable à ses activités d'intermédiaire. Le rédacteur en chef de son journal lui laisse beaucoup de liberté pour cette action culturelle transnationale. Erwin Jaeckle sait bien que la situation économique de Die Tat, quotidien dépendant financièrement des subventions de la Migros, invite ses collaborateurs à se réserver d'autres possibilités professionnelles ${ }^{(34)}$.

Tout comme le correspondant du journal zurichois à Paris, Herbert Lüthy, Allemann devient contributeur régulier d'un réseau de revues internationales englobant les magazines Der Monat (Berlin), Preuves (Paris) et Encounter (Londres). Ce réseau gravitait autour d'un organisme fondé en 1950 et domicilié à Paris, le Congress for Cultural Freedom (CCF) dont on sait aujourd'hui qu'il a obtenu des fonds importants de l'agence de renseignement américaine CIA pour financer ses nombreuses activités dirigées contre l'emprise intellectuelle du communisme ${ }^{(35)}$. Il s'agit donc d'un épisode de la guerre froide contre l'influence soviétique en Europe occidentale, certes intellectuellement fructueux si l'on tient compte de la productivité importante des auteurs rattachés au réseau. À partir de 1953, l'éditeur de Der Monat, Melvin Lasky (1920-2004), accorde une place importante aux commentaires qu'Allemann consacre à la vie politique de la République de Bonn ${ }^{(36)}$. Les contributions d'un «étranger de langue allemande» qui jette un regard compréhensif sur la jeune République fédérale, recouvrant sa souveraineté pleine et entière entre 1952 et 1955, sont particulièrement appréciées par les Américains qui défendent l'hypothèse de la viabilité du nouvel État ouest-allemand contre des voix qui prétendent le contraire, en Allemagne même et à l'étranger ${ }^{(37)}$. Début 1955, Allemann publie dans Der Monat un article où il détaille les différences entre les deux Républiques de Weimar et de Bonn pour souligner la capacité de l'État ouest-allemand de résoudre les problèmes qui vont se poser ${ }^{(38)}$. Lors

34 Voir la lettre que Jaeckle a envoyée à Allemann à la date du 22 août 1979 (BU Bâle, NL 304, A 43, 1-7) où il écrit, entre autres: «Sie haben mich immer so überzeugt, dass ich Ihnen gerne Ihren Wuchs liess und wo es anging förderte. Das ist weniger verdienstlich als einsichtig.»

35 Pierre Grémoin, Intelligence de l'anticommunisme. Le Congrès pour la liberté de la culture à Paris, 1950-1975, Paris, Fayard, 1995; Frances Stonor SAunders, Qui mène la danse? La CIA et la guerre froide culturelle, Paris, Denoël, 2003; Ulrike ACKermann, Sündenfall der Intellektuellen. Ein deutschfranzösischer Streit von 1945 bis heute, Stuttgart, Klett-Cotta, 2003.

36 Pour la stratégie de Lasky, Michael Hochgeschwender, Freiheit in der Offensive? Der Kongreß für kulturelle Freiheit und die Deutschen, Munich, Oldenbourg, 1998, p. 139-158. L'objectif de Lasky qui avait fondé la revue Monat en 1948 à Berlin-Ouest était, selon cette étude, la «aufklärungsorientierte Europäisierung Deutschlands». Ibid., p. 191. Le premier article d'Allemann paraît en janvier 1953: «Das deutsche Parteiensystem. Eine politische Analyse», Der Monat, 5 (1952/53), p. 365-388.

37 Allemann indique lui-même que sa vérité sur l'Allemagne était celle d'un «étranger de langue allemande»: Fritz René Allemann, Bonn ist nicht Weimar, Cologne, Kiepenheuer \& Witsch, 1956, p. 10.

«Bonn ist nicht Weimar», Der Monat, 7 (1954/55), p. 333-341. 
du congrès du CCF à Milan, en septembre 1955, auquel il assiste, le journaliste suisse de Bonn est encouragé par ses interlocuteurs de thématiser cette question dans un livre ${ }^{(39)}$. Fritz René Allemann rédige alors son ouvrage Bonn ist nicht Weimar qui paraîtra en 1956 chez Kiepenheuer \& Witsch à Cologne et où il présente les avantages du système politique de «Bonn» qui a tiré toutes les conclusions de l'échec de la République de Weimar ${ }^{(40)}$. Les critiques universitaires soulignent qu'il s'agit d'un portrait cohérent dressé par «un ami voisin» de l'Allemagne de l'Ouest, portrait qui tient le juste milieu entre la proximité et la distance ${ }^{(41)}$. La presse allemande, elle, met l'accent sur la distanciation. Seul un étranger pouvait soulever la question de savoir si Bonn ressemblait à Weimar ${ }^{(42)}$ !

Pour autant, Allemann n'a pas oublié sa mission initiale d'expliquer la Suisse, sa neutralité, sa démocratie directe et son fédéralisme, aux voisins allemands. Il est sollicité par de nombreuses rédactions de journaux pour rédiger des analyses de la situation politique et socioéconomique suisses et le rapport du pays aux projets d'intégration européenne ${ }^{(43)}$. Les organismes de formation politique qui se sont développés en RFA dans les années 1950 l'invitent régulièrement à des débats sur l'évolution du système politique de «Bonn» depuis 1948/49, évolution dont il a été un témoin oculaire très attentif. La perspective de l'«ami voisin» est particulièrement appréciée pour permettre à la société ouest-allemande de se faire une idée d'elle-même et pour tirer un bilan provisoire des acquis des gouvernements successifs dirigés par le chancelier Adenauer. D’habitude, les observations d'Allemann se présentent comme les «remarques d'un Suisse sur la démocratie allemande», exposées avec beaucoup

39 Pour le congrès de Milan en 1955, Giles Scotт-Sмiтн, «The Congress for Cultural Freedom, the End of Ideology and the 1955 Milan Conference: 'Defining the Parameters of Discourse' ", Journal of Contemporary History, $\mathrm{n}^{\circ} 37$ (2002), p. 437-455.

40 Le livre a été réédité en l'an 2000: Fritz René Allemann, Bonn ist nicht Weimar, éd. Xenia von Bahder, Francfort-sur-le-Main, R.G. Fischer, 2000.

41 Voir, à titre d'exemple, la recension de Dieter Cunz (1910-69), éminent germaniste à l'université d'État de l'Ohio: «[Fritz René Allemann] is a Swiss journalist who has lived in post-war Germany for almost ten years. Speaking the language of the people, he is better equipped than most foreign observers to feel the collective pulse of the German people. Yet, as a foreigner, he is sufficiently removed from the emotional entanglements; it is easier for him to appraise the merits and shortcomings of the new regime than for any native German. From this vantage point of the friendly neighbor wo looks over the fence, not blind to the faults yet appreciative of the improvements, he has written an extremely informative, penetrating, and discerning study, unsurpassed by anything published so far about the Bonn republic. » Dieter Cunz, "Fritz René Allemann: Bonn ist nicht Weimar », The Journal of Politics, n² 20 (1958), p. 596-598.

42 «Alleman [sic!] ist Schweizer und seit sieben [!] Jahren Korrespondent der Zürcher Zeitung 'Die Tat' in Bonn. Während dieser Zeit hat er sich so sehr in die deutsche Materie eingearbeitet, daß ihm seine Kenntnisse schon den Ruf bei seinen Landsleuten eingetragen haben, er sei mehr Deutscher als Schweizer. Diese Ansicht schießt weit über das Ziel hinaus. Das wird jedem bei der Lektüre seines Buches deutlich. Allein schon der Titel weist das aus. Niemand, es sei denn ein Ausländer, könnte die Frage aufwerfen, ob Bonn vielleicht so sei wie Weimar. "Supplément "Das politische Buch", Allgemeine Zeitung Mainz (26.06.1958), BU Bâle, NL 304, H 9.

43 Un recueil d'articles consacrés à la Suisse, pour la période 1952-1991, se trouve dans BU Bâle, NL 304, Db 21. Le premier article paraît à la date du 12.08.1952 dans les Aachener Nachrichten, intitulé «Miniatur-Europa im Dilemma. Schweizer Eidgenossen zwischen Neutralität und europäischer Einheit - Beispiel einer nüchternen Politik». 
de modestie ${ }^{(44)}$. Paradoxalement, cette mise en perspective depuis l'étranger proche devient aussi le signe distinctif et la spécialité d'Allemann dans le paysage médiatique suisse. Son propre journal, Die Tat, lui demande, à partir de 1959, de faire le portrait des cantons suisses, la position du journaliste dans un pays voisin lui facilitant la tâche d'analyser les structures complexes de la Confédération avec recul et objectivité ${ }^{(45)}$. Ce travail, entrepris avec un certain enthousiasme, fait de Fritz René Allemann un avocat fervent du particularisme alémanique et un chantre du fédéralisme helvétique. Ses prises de position en faveur de la segmentation politique et du micro-État cantonal déconcertent plus d'un auditeur en Allemagne. Lorsque le CCF organise à Cologne, le 14 janvier 1960, un débat sur le sujet "La patrie Europe ou l'Europe des patries", en écho à la politique européenne du président de la République Charles de Gaulle en France, Allemann chante les louanges du fédéralisme helvétique, présenté par lui comme un modèle pour l'organisation de l'Europe. À la suite de cette manifestation, son éditeur Johann Caspar Witsch, très impliqué dans les activités du CCF, se moque dans une lettre adressée à l'écrivain Manès Sperber de ces excès de «cantonalisme " ${ }^{(46)}$. La disproportion germano-suisse étant tout d'abord, comme le montre très bien cet exemple, celle entre les grandes et les petites entités politiques. L'incompréhension allemande pour son sujet ne découragera pas Fritz René Allemann qui, en 1965, publiera son "panorama d'une Confédération» sous forme d'un livre qui connaîtra un certain succès, en Allemagne et en Suisse, et qui comprend des essais sur chacun des 25 (aujourd'hui 26) cantons ${ }^{(47)}$.

\section{Rétrospective et perspectives: deux voisins qui se parlent (trop) peu}

La tâche de l'acteur transnational qu'était Fritz René Allemann s'est successivement transformée, en tenant compte de la demande du marché médiatique. Celui qui, en 1951, avait l'ambition d'être un «traducteur» au service du transfert culturel et de la compréhension mutuelle finit par être confiné au rôle de l'«ami voisin» dont l'Allemagne fédérale et la Suisse ont besoin pour une mise en perspective de leur situation depuis un point de vue situé dans l'étranger proche, liant l'intimité à la distance modérément critique. Le journaliste germano-suisse est devenu, vers 1960, le spécialiste de la «petite distanciation» qui accompagne avec bienveillance les autoréflexions nationales. Tout en restant un collaborateur de Die Tat, Allemann déménage de Bonn à Berlin, en 1960, pour y prendre la place de Melvin Lasky, parti pour Londres, à la tête de la rédaction de la revue Der Monat ${ }^{(48)}$. Le départ d'Allemann s'inscrit, d'une manière tout à fait fortuite, dans un tournant dans les relations germano-suisses qui ont connu tout au long des années 1950 un lent retour à la normale de rapports bilatéraux, sans

44 Voir l'intitulé d’une conférence tenue le 24 juin 1960 à l’Académie évangélique de Loccum, en BasseSaxe: «Anmerkungen eines Schweizers zur deutschen Demokratie», BU Bâle, NL 304, F 7.

«Porträts von Schweizer Kantonen. Artikelserie der TAT (1959-63)», BU Bâle, NL 304, Db 22.

«... und Allemann gab eine reizende Hymne auf die schweizer Kantönli». Frank Möller, Das Buch Witsch. Das schwindelerregende Leben des Verlegers Joseph Caspar Witsch, Cologne, Kiepenheuer \& Witsch, 2014, p. 468.

47 Fritz René Allemann, 25 mal die Schweiz. Panorama einer Konföderation, Munich/Zurich, Piper, 1965 ( $2^{\text {nde }}$ édition en 1977).

48 F. Möller, Das Buch Witsch (note 46), p. 511-515. 
grande cordialité, allant de pair avec un certain désintéressement réciproque, sauf dans les domaines économique et touristique. En 1960, la Suisse adhère comme membre fondateur à l'Association européenne de libre-échange (AELE) ${ }^{(49)}$, lancée par le gouvernement britannique pour contrebalancer la Communauté économique européenne (CEE), créée par les traités de Rome de 1957 pour encadrer l'intégration économique et finalement politique du continent, à partir d'un noyau franco-(ouest)allemand. Il s'agit d'un choix politique important pour la Confédération qui l'éloignera des stratégies européennes des pays voisins et notamment de l'Allemagne. Ces évolutions vont plutôt dans le sens d'une aliénation que d'un rapprochement entre les voisins de part et d'autre du Rhin supérieur. En Suisse, la période des passeurs de frontière passionnés, à l'instar d'Erwin Jaeckle ou de Fritz René Allemann, est définitivement révolue. Contrairement aux attentes du germaniste et rédacteur en chef Jaeckle, l'espace public germano-suisse, qui aurait pu constituer l'un des points névralgiques d'un paysage de communication transnational européen après les catastrophes de la première moitié du $\mathrm{Xx}^{\mathrm{e}}$ siècle, n'a pas vu le jour. La communication entre Allemands et Suisses alémaniques reste perturbée, entre autres à cause de la présence des dialectes dans la vie publique helvétique qui s'est renforcée depuis l'immédiat après-guerre. D'un point de vue suisse, la question des relations de la Confédération à l'Europe a remplacé la question allemande. Par rapport à l'Allemagne et, plus largement, à l'Europe, la Suisse devient un "pays à contre-courant», selon l'analyse qu'a apportée le perspicace Herbert Lüthy, en $1962^{(50)}$.

\section{Résumé}

Après la Seconde Guerre mondiale, des intellectuels suisses de langue allemande s'engagent pour renouer le dialogue germano-suisse. Ces échanges transnationaux sont portés par les acteurs de la société civile, sans implication directe des instances politiques. Le journal zurichois Die Tat, dirigé par le germaniste Erwin Jaeckle, lance des initiatives en direction de l'Allemagne tout en soutenant la création d'un État ouest-allemand tourné vers l'Europe. En 1949, le quotidien dépêche un correspondant à Bonn pour couvrir l'évolution en République fédérale. Il s'agit de Fritz René Allemann qui jouera un rôle d'intermédiaire entre les paysages médiatiques suisse et allemand. Cependant, le projet initial d'Allemann, un véritable concept transnational, se réduit vers 1960 à une simple mise en perspective de politiques nationales.

\section{Zusammenfassung}

Nach dem Zweiten Weltkrieg versuchten deutschschweizerische Intellektuelle, den Dialog mit den Deutschen wieder in Gang zu bringen. Diese transnationalen Initiativen gingen von Akteuren der Zivilgesellschaft aus, nicht von den Behörden. Die Zürcher Tageszeitung Die Tat unter der Leitung des Germanisten Erwin Jaeckle hat

49 Roland Maurhofer, Die Schweizerische Europapolitik vom Marshallplan zur EFTA 1947 bis 1960 , Berne, Paul Haupt, 2001 (thèse de l’Université de Saint-Gall).

50 Herbert Lüthy, "La Suisse à contre-courant», Preuves, n 140 (1962), p. 16-28. Voir aussi ID., Die Schweiz als Antithese, Zurich, Arche, 1969. 
sich an diesen Versuchen einer deutsch-schweizerischen Annäherung aktiv beteiligt. Bereits 1949 sandte das Blatt einen ständigen Korrespondenten nach Bonn, um über das politische Leben in der jungen Bundesrepublik zu berichten. Der Journalist Fritz René Allemann spielte dabei zeitweilig die Rolle eines Kulturvermittlers zwischen den beiden Ländern. Sein ursprüngliches transnationales Konzept entwickelte sich jedoch um 1960 hin zu einer lediglich vergleichenden Betrachtung der politischen Vorgänge in der Schweiz und in (West-)Deutschland.

\begin{abstract}
After the Second World War German-speaking Swiss writers and journalists tried to revitalize the intellectual exchange between Switzerland and Germany. These transnational initiatives emanated from the civil society, not from official authorities. A Zurichbased newspaper, Die Tat, managed by Erwin Jaeckle, Swiss author and German scholar, took measures to bring the Swiss society closer to the realities in Germany. In 1949 it sent a correspondent to the new West-German capital Bonn in order to cover the political life in the FRG. This journalist, Fritz René Allemann, played a role as mediator between the societies in the two countries. Nevertheless, around 1960 his originally transnational concept turned into a simple bilateral view on two distinct national politics.
\end{abstract}

